\title{
The Effects of Rotation and Salt Concentration on Thermal Convection in a Linear Magneto-Fluid Layer Overlying a Porous Layer
}

\author{
Maram S. Al-Qurashi ${ }^{*}$, Abdul-Fattah K. Bukhari ${ }^{2}$ \\ ${ }^{1}$ Mathematics Department, College of Sciences, Taif University, Taif, Saudi Arabia; ${ }^{2}$ Mathematics Department, College of Applied \\ Sciences, Umm Al-Qura University, Mecca, Saudi Arbia. \\ Email: *mm.q777@yahoo.com
}

Received June 29 ${ }^{\text {th }}, 2012$; revised July $30^{\text {th }}, 2012$; accepted August $15^{\text {th }}, 2012$

\begin{abstract}
A linear stability analysis is applied to a system consisting of a linear magneto-fluid layer overlying a porous layer affected by rotation and salt concentration on both layers. The flow in the fluid layer is governed by Navier-Stokes's equations and while governed by Darcy-Brinkman's law in the porous medium. Numerical solutions are obtained using Legendre polynomials. These solutions are studied through two modes of instability: stationary instability and overstability when the heat and the salt concentration are effected from above and below.
\end{abstract}

Keywords: Navier-Stokes Equation; Darcy-Brinkman Law; Legendre Polynomials; Salt Concentration; Vertical Linear Magnetic Field

\section{Motivations and Goals}

Thermal instability theory has attracted considerable interest and has been recognized as a problem of fundamental importance in many fields of fluid dynamics. The earliest experiments to demonstrate the onset of thermal instability in fluids are those of Bernard's [1,2]. Benard worked with very thin layers of an incompressible viscous fluid standing on a levelled metallic plate maintained at a constant temperature. The upper surface which was usually free and, being in contact with the air, was at a lower temperature. In his experiments, Benard deduced that a certain critical adverse temperature gradient must be exceeded before instability can set in. The instability of a layer of fluid heated from below and subjected to Coriolis forces has been studied by Chandrasekhar $[3,4]$ for a stationary and overstability case. He showed that the presence of these forces usually has the effect of inhibiting the onset of thermal convection. Nield [5] considered the onset of salt-finger convection in a porous layer. Taunton et al. [6] considered the thermohaline instability and salt-finger in a porous medium and solved the boundary value problem. Sun [7] was the first to consider such a problem, and he used a shooting method to solve the linear stability equations. Sun [7] and Nield [8] used Darcy's law in formulating the equations of porous layer. In Darcy's law of motion in porous

\footnotetext{
${ }^{*}$ Corresponding author.
}

mediums, the Darcy resistance term took the place of the Navier-stokes viscosity term, while in the modified Darcy's law (Brinkman model), suggested by Brinkman [9], the Navier- stokes viscosity term still exists. Chen \& Chen [10] considered the multi-layer problem when the above layer is heated and salted from above, and the solution of the problem is obtained using a shooting method. Lindsay \& Ogden [11] worked in the implementation of spectral methods resistant to the generation of spurious eigenvalues. Lamb [12] used expansion of Chebyshev polynomials to investigate an eigenvalue problem arising from a model discussing a finitely conducting inner core of the earth on magnetically driven instability. Bukhari [13] studied the effects of surface-tension in a layer of conducting fluid with an imposed magnetic field and obtained results when the free surface is deformable and non-deformable. He solved that by using Chebyshev spectral method, and thus obtained some different results from that of Chen \& Chen [10]. Straughan [14] studied the thermal convection in fluid layer overlying a porous layer and considered the problem of lower layer heated from below and surface tension driven on the free top boundary of upper layer. In [15], he also dealt with the same problem considering the ratio depth of the relative layer and investigated the effect of the variation of relevant fluid and porous material properties. Allehiany [16] studied Benard convection in a horizontal porous layer 
permeated by a conducting fluid in the presence of magnetic field and coriolis forces. In this work, we studied the effects of rotation and salt concentration on thermal convection in a linear magneto-fluid overlying a porous layer. The numerical solution was presented in different boundary conditions solved by using Legendre polynomials.

\section{The Governing Equations}

We consider a fluid layer overlying a porous layer so that the top of the porous layer touches the bottom of the fluid layer. The plane interface between the two layers is $x_{3}=0$, the upper boundary of the fluid layer is $x_{3}=d_{f}$ and the lower boundary of the porous medium layer is $x_{3}=-d_{m}$ where the subscripts $f$ and $\mathrm{m}$ denote the fluid layer and porous medium layer respectively. We suppose that the upper layer is filled with an incompressible thermally and electrically conducting viscous fluid consisting of melted salt which flows in it and governed by Navier-Stokes equations. However, the lower layer is occupied by a porous medium permeated by the fluid flowing in it and governed by Darcy-Brinkman's law. Both layers subjected to a constant vertical linear magnetic field and affected by a rotation around $x_{3}$ with a constant angular velocity $\Omega$. Gravity g acts in the negative direction of $x_{3}$ (see Figure 1).

Convection is driven by the temperature depending on the fluid density and salting, and damped by viscosity. The Oberbeck-Boussineq approximation is used as the density of fluid is constant everywhere except in the body force term where the density is linearly proportional to temperature and salt concentration, i.e.

$$
\rho_{f}=\rho_{0}\left[1-\alpha\left(T-T_{0}\right)+\beta\left(S-S_{0}\right)\right] .
$$

where $T$ denotes the Kelvin temperature of the fluid, $S$ is the salt concentration, $\rho_{0}$ is the density of fluid at $T_{0}$ and $S_{0}, \alpha$ (constant) is the thermal coefficient of volume expansion of the fluid and $\beta$ (constant) is the salting coefficient of volume expansion of the fluid. Let $V$ be the solenoidal velocity of the fluid.

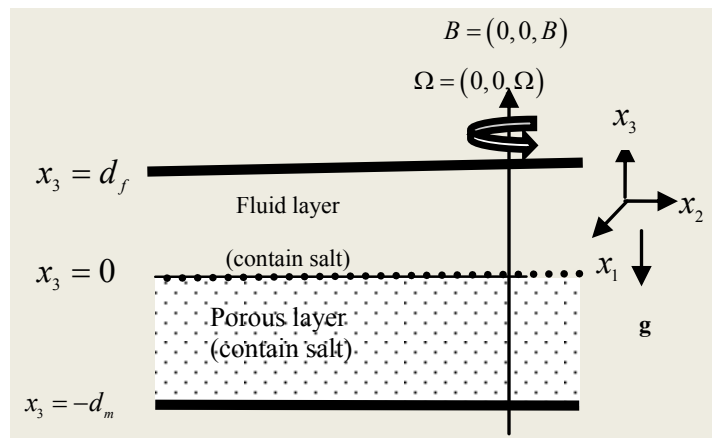

Figure 1. Schematic diagram of the problem.
Let $V, H, B, J$ and $E$ be respectively the solenoiddal velocity of the fluid, the magnetic field, the magnetic induction, the current density and the electric field. Hence

$$
\operatorname{div} V=0, \operatorname{div} B=0
$$

$H, B, J$ and $E$ connected by the relations

$$
B=\mu_{m} H, J=\bar{\sigma}[E+(V \times B)]
$$

where $\mu_{m}$ is the magnetic permeability and $\bar{\sigma}$ is the electrical conductivity. And the Maxwell equations

$$
\operatorname{curl} E=-\frac{\partial B}{\partial \mathrm{t}}, J=\operatorname{curl} H
$$

where the displacement current has been neglected in the second of these Maxwell equations as is customary in situation when free charge is instantaneously dispersed. By substituting from (3), (4) $)_{2}$ in to (4) obtain by

$$
\begin{aligned}
\frac{\partial B}{\partial \mathrm{t}} & =-\operatorname{curl}\left[\frac{1}{\bar{\sigma}} J-(V \times B)\right] \\
& =-\operatorname{curl}\left[\frac{1}{\mu_{m} \bar{\sigma}} \operatorname{curl} B-(V \times B)\right] \\
& =\operatorname{curl}(V \times B)-\eta \operatorname{curl} \operatorname{curl} B
\end{aligned}
$$

where $\eta=\left(\frac{1}{\mu_{m} \bar{\sigma}}\right)$ is electrical resistivity. By using

$$
\text { curl curl } B=\operatorname{grad} \operatorname{div}(B)-\nabla^{2}(B)
$$

and

$$
\operatorname{curl}(V \times B)=(B \cdot \nabla) V-(V \cdot \nabla) B
$$

then Equation (5) reduce to

$$
\frac{\partial B}{\partial \mathrm{t}}=(B \cdot \nabla) V-(V \cdot \nabla) B+\eta \nabla^{2} B
$$

The equation of motion is

$$
\begin{aligned}
& \rho_{0}\left(\frac{\partial V}{\partial \mathrm{t}}+V \cdot \nabla V\right) \\
= & -\nabla \bar{P}+\mu \nabla^{2} V+\rho g+\rho_{0}(V \times \Omega)+J \times B
\end{aligned}
$$

where $\bar{P}$ is hydrostatic pressure, $\mu$ is the dynamic viscosity and $\nabla^{2}$ is three-dimensional Laplacian operator. And by subsituting from $(3)_{1},(4)_{2}$ in to the Lorentz force $J \times B$ we obtain

$$
\begin{aligned}
J \times B & =\frac{1}{\mu_{m}} \operatorname{curl} B \times B \\
& =\frac{1}{\mu_{m}}\left(B \cdot \nabla B-\frac{1}{2} \nabla B^{2}\right)
\end{aligned}
$$

Hence the equation of motion becomes 


$$
\begin{aligned}
\rho_{0}\left(\frac{\partial V}{\partial \mathrm{t}}+V \cdot \nabla V\right)= & -\nabla\left(\bar{P}+\frac{1}{8 \pi \mu_{m}} \nabla B^{2}\right)+\mu \nabla^{2} V_{f} \\
& +\rho g+2 \rho_{0}(V \times \Omega)+\frac{1}{\mu_{m}} B \cdot \nabla B
\end{aligned}
$$

and so the governing equations of the fluid layer are

$$
\begin{aligned}
& {\left[\frac{\partial V_{f}}{\partial t}+V_{f} \cdot \nabla V_{f}\right]} \\
& =-\nabla \frac{P_{f}}{\rho_{0}}+v \nabla^{2} V_{f} \\
& -g\left[1-\alpha\left(T_{f}-T_{0}\right)+\beta\left(S_{f}-S_{0}\right)\right] \\
& +2\left(V_{f} \times \Omega_{f}\right)+\frac{1}{\rho_{0} \mu_{m f}}\left(B_{f} \cdot \nabla B_{f}\right), \\
& \left(\rho_{0} c_{p}\right)_{f}\left[\frac{\partial T_{f}}{\partial t}+V_{f} \cdot \nabla T_{f}\right]=k_{f} \nabla^{2} T_{f} \\
& \frac{\partial S_{f}}{\partial t}+V_{f} \cdot \nabla S_{f}=D_{f} \nabla^{2} S_{f} \\
& J_{f}=\frac{1}{\mu_{m f}} \operatorname{curl} B_{f} \\
& \frac{\partial B_{f}}{\partial t}=\left(B_{f} \cdot \nabla\right) V_{f}-\left(V_{f} \cdot \nabla\right) B_{f}+\eta_{f} \nabla^{2} B_{f}
\end{aligned}
$$

and the governing equations of the porous medium layer are

$$
\begin{aligned}
& \frac{1}{\varphi} \frac{\partial V_{m}}{\partial t}=-\nabla \frac{P_{m}}{\rho_{0}}-\frac{v}{K} V_{m}+v \nabla^{2} V_{m} \\
& -g\left[1-\alpha\left(T_{m}-T_{0}\right)+\beta\left(S_{m}-S_{0}\right)\right] \\
& +2\left(V_{m} \times \Omega_{m}\right)+\frac{1}{\rho_{0} \mu_{m m}}\left(B_{m} \cdot \nabla B_{m}\right) \\
& \left(\rho_{0} c\right)_{m} \frac{\partial T_{m}}{\partial t}+\left(\rho c_{p}\right)_{f} V_{m} \nabla T_{m}=k_{m} \nabla^{2} T_{m} \\
& \varphi \frac{\partial S_{m}}{\partial t}+V_{m} \cdot \nabla S_{m}=D_{m} \nabla^{2} S_{m} \\
& J_{m}=\frac{1}{\mu_{m m}} \operatorname{curl} B_{m} \\
& \frac{\partial B_{m}}{\partial \mathrm{t}}=\left(B_{m} \cdot \nabla\right) V_{m}-\left(V_{m} \cdot \nabla\right) B_{m}+\eta_{m} \nabla^{2} B_{m}
\end{aligned}
$$

where $P_{f}, P_{m}$ are the modified pressure of the fluid and the porous medium layers respectively and
$\frac{1}{2}\left|\Omega_{f} \times r\right|^{2}, \frac{1}{2}\left|\Omega_{m} \times r\right|^{2}$ are the centrifugal force of the fluid and porous medium layer respectively, $V_{f}, V_{m}$ are the solenoidal and seepage velocity respectively, $2\left(V_{f} \times \Omega_{f}\right), 2\left(V_{m} \times \Omega_{m}\right)$ are the coriolis acceleration of the fluid and porous medium layer respectively, $T_{f}, T_{m}$ are the Kelvin temperature of the fluid and porous medium layer respectively, $S_{f}, S_{m}$ are salt concentration of the fluid and porous medium layer respectively, $D_{f}, D_{m}$ are the mass diffusivity of the fluid and porous medium layer respectively, $\mu_{m f}, \mu_{m m}$ are magnetic permeability of fluid and porous layer respectively, $k_{f}, k_{m}$ are the thermal and overall thermal conductivity of fluid and porous layers respectively, $v=\mu / \rho_{0}$ is the kinematic viscosity, $K$ is the permeability, $\phi$ is its porosity and $\left(\rho_{0} c_{p}\right)_{f},\left(\rho_{0} c\right)_{m}$ are the heat and overall heat capacity per unit volume of the fluid and porous medium layers at constant pressure. In fact

$$
\left(\rho_{0} c\right)_{m}=\varphi\left(\rho_{0} c_{p}\right)_{f}+(1-\varphi)\left(\rho_{0} c_{p}\right)_{m}
$$

where $\left(\rho_{0} c_{p}\right)_{m}$ is the heat capacity per unit volume of the porous substrate. Suppose that $x_{3}=d_{f}$ is rigid and maintained at constant temperature $T_{u}$ and constant salt concentration $S_{u}$, and $x_{3}=-d_{m}$ is impenetrable and maintained at constant temperature $T_{l}$ and constant salt concentration $S_{l}$, then the boundary conditions can be written as

$$
\begin{aligned}
& w_{f}\left(d_{f}\right)=0, \frac{\partial w_{f}}{\partial x_{3}}\left(d_{f}\right)=0, \\
& T_{f}\left(d_{f}\right)=T_{u}, S_{f}\left(d_{f}\right)=S_{u} \\
& \zeta_{3_{f}}\left(d_{f}\right)=0, J_{3 f}\left(d_{f}\right)=0 \quad \frac{\partial B_{f}}{\partial x_{3}}\left(d_{f}\right)
\end{aligned}
$$

on the upper boundary, and

$$
\begin{aligned}
& w_{m}\left(-d_{m}\right)=0, \frac{\partial w_{m}}{\partial x_{m}}\left(d_{m}\right)=0, \\
& T_{m}\left(-d_{m}\right)=T_{l}, S_{m}\left(-d_{m}\right)=S_{l} \\
& \zeta_{3_{m}}\left(d_{m}\right)=0, J_{3 m}\left(d_{m}\right)=0 \quad \frac{\partial B_{m}}{\partial x_{3}}\left(d_{m}\right)
\end{aligned}
$$

on the lower boundary, where $w_{f}$ and $w_{m}$ are the normal axial velocity components of the fluid in fluid layer and porous medium layer respectively, $\zeta_{3_{f}}$ and $\zeta_{3_{m}}$ are the normal axial vortecity components of the fluid in. fluid layer and porous medium layer respectively.

The boundary conditions on the interface plane $x_{3}=0$ are based on the assumption that temperature, salt concentration, heat flux, salt flux, normal and tangential fluid velocity, normal stress and tangential stress are continuous so that 


$$
\begin{aligned}
& T_{f}(0)=T_{m}(0), S_{f}(0)=S_{m}(0) \\
& k_{f} \frac{\partial T_{f}}{\partial x_{3}}(0)=k_{m} \frac{\partial T_{m}}{\partial x_{3}}(0), D_{f} \frac{\partial S_{f}}{\partial x_{3}}(0)=D_{m} \frac{\partial S_{m}}{\partial x_{3}}(0) \\
& w_{f}(0)=w_{m}(0), u_{f}(0)=u_{m}(0), v_{f}(0)=v_{m}(0) \\
& -p_{f}(0)+2 \mu \frac{\partial w_{f}}{\partial x_{3}}(0)=-p_{m}(0) 2+\mu \frac{\partial w_{m}}{\partial x_{3}}(0) \\
& \frac{\partial u_{f}}{\partial x_{3}}(0)=\frac{\partial u_{m}}{\partial x_{3}}(0), \frac{\partial v_{f}}{\partial x_{3}}(0)=\frac{\partial v_{m}}{\partial x_{3}}(0) \\
& J_{f}(0)=\frac{\mu_{m f}}{\mu_{m m}} J_{m}(0), \frac{k_{f}}{\mu_{m f}} \frac{\partial J_{f}}{\partial x_{3}}(0)=\frac{k_{m}}{\mu_{m m}} \frac{\partial J_{m}}{\partial x_{3}}(0) \\
& B_{f}(0)=B_{m}(0), k_{f} \frac{\partial B_{f}}{\partial x_{3}}(0)=k_{m} \frac{\partial B_{m}}{\partial x_{3}}(0)
\end{aligned}
$$

Equations (10)-(19) have an equilibrium solution satisfying the boundary conditions (20)-(22) on the form

$$
\begin{aligned}
& V_{f}=0, V_{m}=0, \\
& -\nabla P_{f}+\rho_{f} g=0,-\nabla P_{m}+\rho_{f} g=0, \\
& \nabla^{2} T_{f}=\nabla^{2} T_{m}=0, \nabla^{2} S_{f}=\nabla^{2} S_{m}=0, \\
& \Omega_{f}=\left(0,0, \Omega_{f}\right), \Omega_{f} \text { constant, } \\
& \Omega_{m}=\left(0,0, \Omega_{m}\right), \Omega_{m} \text { constant, } \\
& B_{f}=\left(0,0, B_{f}\right), B_{f} \text { constant, } \\
& B_{m}=\left(0,0, B_{m}\right), B_{m} \text { constant }
\end{aligned}
$$

and with the boundary conditions

$$
\begin{aligned}
& T_{f}\left(d_{f}\right)=T_{u}, T_{m}\left(-d_{m}\right)=T_{l}, \\
& S_{f}\left(d_{f}\right)=S_{u}, S_{m}\left(-d_{m}\right)=S_{l}
\end{aligned}
$$

and the interface conditions

$$
\begin{aligned}
& T_{f}(0)=T_{m}(0), k_{f} \frac{\partial T_{f}}{\partial x_{3}}(0)=k_{m} \frac{\partial T_{m}}{\partial x_{3}}(0), \\
& S_{f}(0)=S_{m}(0), D_{f} \frac{\partial S_{f}}{\partial x_{3}}(0)=D_{m} \frac{\partial S_{m}}{\partial x_{3}}(0), \\
& P_{f}(0)=P_{m}(0)
\end{aligned}
$$

the equilibrium temperature field, hydrostatic pressure and salt concentration in the fluid layer and porous medium layer are respectively

$$
\begin{aligned}
& T_{f}=T_{0}-\left(T_{0}-T_{u}\right) \frac{x_{3}}{d_{f}}, P_{f}=P_{f}\left(x_{3}\right), \\
& S_{f}=S_{0}-\left(S_{0}-S_{u}\right) \frac{x_{3}}{d_{f}}, 0 \leq x_{3} \leq d_{f}, \\
& T_{m}=T_{0}-\left(T_{l}-T_{0}\right) \frac{x_{3}}{d_{m}}, P_{m}=P_{m}\left(x_{3}\right), \\
& S_{m}=S_{0}-\left(S_{l}-S_{0}\right) \frac{x_{3}}{d_{m}},-d_{m} \leq x_{3} \leq 0
\end{aligned}
$$

where $T_{0}=\frac{k_{f} d_{m} T_{u}+k_{m} d_{f} T_{l}}{k_{f} d_{m}+k_{m} d_{f}}, S_{0}=\frac{D_{f} d_{m} S_{u}+D_{m} d_{f} S_{l}}{D_{f} d_{m}+D_{m} d_{f}}$

\section{Perturbed Equations}

We apply the perturbation by following linear perturbation quantities

$$
\begin{aligned}
& V_{f}=0+\varepsilon v_{f}, P_{f}=P_{f}\left(x_{3}\right)+\varepsilon p_{f}, \\
& T_{f}=T_{0}-\left(T_{0}-T_{u}\right) \frac{x_{3}}{d_{f}}+\varepsilon \theta_{f}, \\
& S_{f}=S_{0}-\left(S_{0}-S_{u}\right) \frac{x_{3}}{d_{f}}+\varepsilon s_{f}, \\
& J_{f}=0+\varepsilon j_{f}, B_{f}=B_{f} e_{3}+\varepsilon b_{f}, \\
& V_{m}=0+\varepsilon v_{m}, P_{m}=P_{m}\left(x_{3}\right)+\varepsilon p_{m}, \\
& T_{m}=T_{0}-\left(T_{l}-T_{0}\right) \frac{x_{3}}{d_{m}}+\varepsilon \theta_{m}, \\
& S_{m}=S_{0}-\left(S_{l}-S_{0}\right) \frac{x_{3}}{d_{m}}+\varepsilon S_{m}, \\
& J_{m}=0+\varepsilon j_{m}, B_{m}=B_{m} e_{3}+\varepsilon b_{m},
\end{aligned}
$$

to the governing equations in the fluid layer and porous medium layer respectively and to the boundary conditions. After perturbation, the non-dimensionlisation will be apply by using

$$
\begin{aligned}
& t=\frac{d_{f}^{2}}{\lambda_{f}} \dot{t}_{f}, v_{f}=\frac{v}{d_{f}} v_{f}, \\
& x=d_{f} \dot{x}_{f}, p_{f}=\frac{\rho_{0} v^{2}}{d_{f}^{2}} p_{f}, \\
& s_{f}=\frac{\left|S_{0}-S_{u}\right| v}{D_{f}} \dot{s}_{f}, \theta_{f}=\frac{\left|T_{0}-T_{u}\right| v}{\lambda_{f}} \theta_{f}, \\
& b_{f}=\frac{\rho_{0} \mu_{m f} v^{2}}{B_{f} d_{f}^{2}} b_{f}, j_{f}=\frac{\rho_{0} v^{2}}{B_{f} d_{f}^{3}} j_{f}
\end{aligned}
$$

for the fluid layer, and by using

$$
\begin{aligned}
& x=d_{m} \dot{x}_{m}, v_{m}=\frac{v}{d_{m}} v_{m}^{\cdot}, \\
& t=\frac{d_{m}^{2}}{\lambda_{m}} t_{m}^{\cdot}, p_{m}=\frac{\rho_{0} v^{2}}{K} p_{m}^{\cdot}, \\
& \theta_{m}=\frac{\left|T_{l}-T_{0}\right| v}{\lambda_{m}} \theta_{m}^{\cdot}, s_{m}=\frac{\left|S_{l}-S_{0}\right| v_{m}}{D_{m}} s_{m} \\
& j_{m}=\frac{\rho_{0} v^{2}}{B_{m} d_{m}^{3}} \ddot{j}_{m}, b_{m}=\frac{\rho_{0} \mu_{m m} v^{2}}{B_{m} d_{m}^{2}} b_{m}
\end{aligned}
$$

for the porous medium layer, here $\lambda_{m}=k_{m} /\left(\rho c_{p}\right)_{f}$ and $\lambda_{f}=k_{f} /\left(\rho c_{p}\right)_{f}$ are the thermal diffusivity of the fluid 
phase and porous medium respectively, then the Equations (10)-(14) becomes

$$
\begin{aligned}
& \frac{1}{P_{r_{f}}} \frac{\partial v_{f}}{\partial t_{f}}=-\nabla p_{f}+\nabla^{2} v_{f}+R t_{f} \theta_{f} \\
& \quad-R s_{f} s_{f}+T a_{f}\left(v_{f} \times e_{3}\right)+\frac{\partial b_{f}}{\partial x_{3}} \\
& \frac{\partial \theta_{f}}{\partial t_{f}}+F_{T} v_{f}=\nabla^{2} \theta_{f} \\
& \frac{1}{L e_{f}} \frac{\partial s_{f}}{\partial t_{f}}+F_{S} v_{f}=\nabla^{2} s_{f} \\
& J_{f}=\operatorname{curl} b_{f} \\
& \frac{1}{P_{m_{f}}} \frac{\partial b_{f}}{\partial t_{f}}=Q_{f} \frac{\partial v_{f}}{\partial x_{3}}+\nabla^{2} b_{f}
\end{aligned}
$$

where $e_{3}$ is the unit vector in the $x_{3}$-direction and $P_{r_{f}}, R t_{f}, R s_{f}, T a_{f}, Q_{f}, L e_{f}$ and $P_{m_{f}}$ are non-dimensional numbers denote the viscous Prandtl number, thermal Rayleigh number, salt Rayleigh number, Taylor number, Chandraskhar number, Lewis number and magnetic Prandtl number of the fluid layer and given by

$$
\begin{aligned}
& P_{r_{f}}=\frac{v}{\lambda_{f}}, R t_{f}=\frac{g \alpha\left|T_{0}-T_{u}\right| d_{f}^{3}}{v \lambda_{f}}, R s_{f}=\frac{g \beta\left|S_{0}-S_{u}\right| d_{f}^{3}}{v D_{f}}, \\
& T a_{f}=\frac{2 \Omega_{f} d_{f}^{2}}{v}, Q_{f}=\frac{d_{f}^{2} B_{f}^{2}}{\mu_{m f} \rho_{0} v \eta_{f}}, \\
& L e_{f}=\frac{D_{f}}{\lambda_{f}}, P_{m_{f}}=\frac{\eta_{f}}{\lambda_{f}}
\end{aligned}
$$

and the Equations (15)-(19) becomes

$$
\begin{gathered}
\frac{D a}{\varphi P_{r_{m}}} \frac{\partial v_{m}}{\partial t_{m}}=-\nabla p_{m}-v_{m}+R t_{m} \theta_{m} \\
\quad-R s_{m} s_{m}+T a_{m}\left(v_{m} \times e_{3}\right)+D a \frac{\partial b_{m}}{\partial x_{3}} \\
G_{m} \frac{\partial \theta_{m}}{\partial t_{m}}+F_{T} v_{m}=\nabla^{2} \theta_{m} \\
\frac{\varphi}{L e_{m}} \frac{\partial s_{m}}{\partial t_{m}}+F_{S} v_{m}=\nabla^{2} s_{m} \\
J_{m}=\operatorname{curl} b_{m} \\
\frac{1}{P_{m_{m}}} \frac{\partial b_{m}}{\partial t_{m}}=Q_{m} \frac{\partial v_{m}}{\partial x_{3}}+\nabla^{2} b_{m}
\end{gathered}
$$

where $G_{m}=\left(\rho_{0} c\right)_{m} /\left(\rho_{0} c_{p}\right)_{f}$ and $D a, P_{r m}, R t_{m}, R s_{m}, T a_{m}$, $Q_{m}, L e_{m}$ and $P_{m_{m}}$ are non-dimensional numbers denote the Darcy number, viscous Prandtl number, thermal Rayleigh number, salt Rayleigh number, Taylor number,
Chandarsekhar number, Lewis number and magnetic Prandtl number of the porous medium layer and given by

$$
\begin{aligned}
& D a=\frac{K}{d_{m}^{2}}, P_{r_{m}}=\frac{v}{\lambda_{m}}, R t_{m}=\frac{g \alpha\left|T_{l}-T_{0}\right| K d_{m}}{v \lambda_{m}}, \\
& R s_{m}=\frac{g \beta\left|S_{l}-S_{0}\right| K d_{m}}{v D_{m}}, T a_{m}=\frac{2 \Omega_{m} K}{v}, \\
& Q_{m}=\frac{d_{m}^{2} B_{m}^{2}}{\mu_{m m} \rho_{0} v \eta_{m}}, L e_{m}=\frac{D_{m}}{\lambda_{m}}, P_{m_{m}}=\frac{\eta_{m}}{\lambda_{m}}
\end{aligned}
$$

and where

$$
\begin{aligned}
& F_{T}=\frac{-\left(T_{0}-T_{u}\right)}{\left|T_{0}-T_{u}\right|}=\frac{-\left(T_{l}-T_{0}\right)}{\left|T_{l}-T_{0}\right|} \\
& =\left\{\begin{array}{c}
-1 \text {, when heating from below, } \\
1, \text { when heating from above, }
\end{array}\right. \\
& F_{S}=\frac{-\left(S_{0}-S_{u}\right)}{\left|S_{0}-S_{u}\right|}=\frac{-\left(S_{l}-S_{0}\right)}{\left|S_{l}-S_{0}\right|} \\
& =\left\{\begin{array}{l}
-1, \text { when salt concentration from below, } \\
1, \text { when salt concentration from above. }
\end{array}\right.
\end{aligned}
$$

The boundary conditions (20)-(22) becomes

$$
\begin{aligned}
& w_{f}(1)=0, \frac{\partial w_{f}}{\partial x_{3}}(1)=0, \theta_{f}(1)=0, \\
& s_{f}(1)=0, \xi_{3_{f}}(1)=0, \\
& \frac{\partial b_{f}}{\partial x_{3}}(1)=0, j_{3_{f}}(1)=0, \\
& \frac{\partial \theta_{f}}{\partial x_{3}}(0)=\varepsilon_{T} \frac{\partial \theta_{m}}{\partial x_{3}}(0), \frac{\partial s_{f}}{\partial x_{3}}(0)=\varepsilon_{S} \frac{\partial s_{m}}{\partial x_{3}}(0), \\
& w_{f}(0)=\hat{d} w_{m}(0), u_{f}(0)=\hat{d} u_{m}(0), v_{f}(0)=\hat{d} v_{m}(0), \\
& b_{f}(0)=\frac{\hat{d}}{\hat{m}} b_{m}(0), \frac{\partial b_{f}}{\partial x_{3}}(0)=\frac{\hat{d}^{3}}{\hat{m}_{T}} \frac{\partial b_{m}}{\partial x_{3}}(0), \\
& j_{f}(0)=\frac{\hat{d}^{2}}{\hat{m}} j_{m}(0), \frac{\partial j_{f}}{\partial x_{3}}(0)=\frac{\hat{d}^{3}}{\hat{m}_{\varepsilon_{T}}} \frac{\partial j_{f}}{\partial x_{3}}(0), \\
& p_{f}(0)-2 \frac{\partial w_{f}}{\partial x_{3}}(0)=\frac{\hat{d}^{2}}{D a} p_{m}(0)-2 \hat{d}^{2} \frac{\partial w_{m}}{\partial x_{3}}(0), \\
& \frac{\partial u_{f}}{\partial x_{3}}(0)=\hat{d}^{2} \frac{\partial u_{f}}{\partial x_{3}}(0), \frac{\partial v_{f}}{\partial x_{3}}(0)=\hat{d}^{2} \frac{\partial v_{f}}{\partial x_{3}} \\
& w_{m}(-1)=0, \frac{\partial w_{m}}{\partial x_{3}}(-1)=0, \\
& \theta_{m}(-1)=0, s_{m}(-1)=0, \xi_{3_{m}}(-1)=0, \\
& \frac{\partial b_{m}}{\partial x_{3}}(1)=0, j_{3_{m}}(1)=0
\end{aligned}
$$


where $\hat{d}, \hat{n}, \hat{m}, \varepsilon_{T}, \varepsilon_{S}, \gamma_{T}$ and are given by

$$
\begin{aligned}
\hat{d} & =\frac{d_{f}}{d_{m}}, \hat{n}=\frac{\Omega_{f}}{\Omega_{m}}, \hat{m}=\frac{B_{m}}{B_{f}} \\
\gamma_{T} & =\frac{\left|T_{0}-T_{u}\right|}{\left|T_{l}-T_{0}\right|}=\frac{\hat{d}}{\varepsilon_{T}}, \gamma_{S}=\frac{\left|S_{0}-S_{u}\right|}{\left|S_{l}-S_{0}\right|}=\frac{\hat{d}}{\varepsilon_{S}}, \\
\varepsilon_{T} & =\frac{\lambda_{f}}{\lambda_{m}}, \varepsilon_{S}=\frac{D_{f}}{D_{m}},
\end{aligned}
$$

and

$$
\begin{aligned}
& P_{r_{f}}=\frac{1}{\varepsilon_{T}} P_{r_{m}}, R t_{f}=\frac{\hat{d}^{4}}{\varepsilon_{T}^{2} D a} R t_{m}, R s_{f}=\frac{\hat{d}^{4}}{\varepsilon_{S}^{2} D a} R s_{m}, \\
& T a_{f}=\frac{\hat{n} \hat{d}^{2}}{D a} T a_{m}, L e_{f}=\frac{\varepsilon_{S}}{\varepsilon_{T}} L e_{m} .
\end{aligned}
$$

\section{The Linearized Equations}

Linearization will be done by neglecting all products and powers (higher than the first) of the linear perturbation quantity, and by dropping the $(\bullet)$ superscript, then by taking the curl of the Equations (30) and (35) we obtain

$$
\begin{gathered}
\frac{1}{P_{r_{f}}} \frac{\partial \xi_{f}}{\partial t_{f}}=\nabla^{2} \xi_{f}+R t_{f}\left(\nabla \times \theta_{f}\right) \\
-R s_{f}\left(\nabla \times s_{f}\right)+T a_{f} \frac{\partial v_{f}}{\partial x_{3}}+\frac{\partial J_{f}}{\partial x_{3}} \\
\frac{D a}{\varphi P_{r_{m}}} \frac{\partial \xi_{m}}{\partial t_{m}}=-\xi_{m}+D a \nabla^{2} \xi_{m}+R t_{m}\left(\nabla \times \theta_{m}\right) \\
-R s_{m}\left(\nabla \times s_{m}\right)+T a_{m} \frac{\partial v_{m}}{\partial x_{3}}+D a \frac{\partial J_{m}}{\partial x_{3}}
\end{gathered}
$$

if return to the original Equations (30) and (35), but in this case we take the (curl curl). Thus

$$
\begin{aligned}
& \frac{1}{P_{r_{f}}} \frac{\partial}{\partial t_{f}} \nabla^{2} v_{f} \\
& =\nabla^{4} v_{f}-R t_{f}\left(\nabla \frac{\partial \theta_{f}}{\partial x_{3}}-\nabla^{2} \theta_{f} e_{3}\right) \\
& +R s_{f}\left(\nabla \frac{\partial s_{f}}{\partial x_{3}}-\nabla^{2} s_{f} e_{3}\right)-T a_{f} \frac{\partial \xi_{f}}{\partial x_{3}}+\nabla^{2} \frac{\partial b_{f}}{\partial x_{3}} \\
& \frac{D a}{\varphi P_{r_{m}}} \frac{\partial}{\partial t_{m}} \nabla^{2} v_{m} \\
& =\nabla^{2} v_{m}+D a \nabla^{4} v_{m}-R t_{m}\left(\nabla \frac{\partial \theta_{m}}{\partial x_{3}}-\nabla^{2} \theta_{m} e_{3}\right) \\
& +R s_{m}\left(\nabla \frac{\partial s_{m}}{\partial x_{3}}-\nabla^{2} s_{m} e_{3}\right)-T a_{m} \frac{\partial \xi_{m}}{\partial x_{3}}+D a \nabla^{2} \frac{\partial b_{m}}{\partial x_{3}}
\end{aligned}
$$

and if we use the curl of Equations (34) and (39) with using (33) and (38), we obtain

$$
\begin{aligned}
& \frac{1}{P_{m_{f}}} \frac{\partial J_{f}}{\partial t_{f}}=Q_{f} \frac{\partial \xi_{f}}{\partial x_{3}}+\nabla^{2} J_{f} \\
& \frac{1}{P_{m_{m}}} \frac{\partial J_{m}}{\partial t_{m}}=Q_{m} \frac{\partial \xi_{m}}{\partial x_{3}}+\nabla^{2} J_{m}
\end{aligned}
$$

Now, the third components of Equations (31), (32), (34), (36), (37), (39) and (41)-(46) are

$$
\begin{aligned}
& \frac{1}{P_{r_{f}}} \frac{\partial \xi_{3_{f}}}{\partial t_{f}}=\nabla^{2} \xi_{3_{f}}+T a_{f} \frac{\partial w_{f}}{\partial x_{3}}+\frac{\partial j_{3_{f}}}{\partial x_{3}} \\
& \frac{1}{P_{r_{f}}} \frac{\partial}{\partial t_{f}} \nabla^{2} w_{f} \\
& =\nabla^{4} w_{f}+R t_{f} \nabla_{2}^{2} \theta_{f} \\
& -R s_{f} \nabla_{2}^{2} s_{f}-T a_{f} \frac{\partial \xi_{3_{f}}}{\partial x_{3}}+\nabla^{2} \frac{\partial b_{f}}{\partial x_{3}} \\
& \frac{\partial \theta_{f}}{\partial t_{f}}+F_{T} w_{f}=\nabla^{2} \theta_{f} \\
& \frac{1}{L e_{f}} \frac{\partial s_{f}}{\partial t_{f}}+F_{S} w_{f}=\nabla^{2} s_{f} \\
& \frac{1}{P_{m_{f}}} \frac{\partial b_{f}}{\partial t_{f}}=Q_{f} \frac{\partial w_{f}}{\partial x_{3}}+\nabla^{2} b_{f} \\
& \frac{1}{P_{m_{f}}} \frac{\partial j_{3_{f}}}{\partial t_{f}}=Q_{f} \frac{\partial \xi_{3_{f}}}{\partial x_{3}}+\nabla^{2} j_{3_{f}} \\
& \frac{D a}{\varphi P_{r_{m}}} \frac{\partial \xi_{3_{m}}}{\partial t_{m}}=-\xi_{3 m}+D a \nabla^{2} \xi_{3 f} \\
& +T a_{m} \frac{\partial w_{m}}{\partial x_{3}}+D a \frac{\partial j_{3_{m}}}{\partial x_{3}} \\
& \frac{D a}{\varphi P_{r_{m}}} \frac{\partial}{\partial t_{m}} \nabla^{2} w_{m} \\
& =-\nabla^{2} w_{m}+D a \nabla^{4} w_{m}+R t_{m} \nabla_{2}^{2} \theta_{m} \\
& -R s_{m} \nabla_{2}^{2} s_{m}-T a_{m} \frac{\partial \xi_{3 m}}{\partial x_{3}}+D a \nabla^{2} \frac{\partial b_{f m}}{\partial x_{3}} \\
& G_{m} \frac{\partial \theta_{m}}{\partial t_{m}}+F_{T} w_{m}=\nabla^{2} \theta_{m} \\
& \frac{\varphi}{L e_{m}} \frac{\partial s_{m}}{\partial t_{m}}+F_{S} w_{m}=\nabla^{2} s_{m} \\
& \frac{1}{P_{m_{f n}}} \frac{\partial j_{3_{m f}}}{\partial t_{m}}=Q_{f} \frac{\partial \xi_{3_{f}}}{\partial x_{3}}+\nabla^{2} j_{3_{m f}}
\end{aligned}
$$




$$
\frac{1}{P_{m_{m}}} \frac{\partial b_{m}}{\partial t_{m}}=Q_{f} \frac{\partial w_{m}}{\partial x_{m}}+\nabla^{2} b_{m}
$$

where $\nabla_{2}^{2}=\nabla^{2}-\frac{\partial^{2}}{\partial x_{3}^{2}}$ is tow-dimensional Laplacian operator and $\nabla^{4}=\left(\nabla^{2}\right)^{2}$. We apply the normal modes solution in the form

$$
\Phi(x, t)=\Phi\left(x_{3}\right) \exp \left[i\left(n x_{1}+m x_{2}\right)+\sigma t\right]
$$

with the functions $w_{f}, \theta_{f}, s_{f}, \xi_{f}, j_{f}, b_{f}, w_{m}, \theta_{m}, s_{m}, \xi_{m}$, $j_{m}$ and $b_{m}$. Thus the governing equations are

$$
\begin{aligned}
& \frac{\sigma_{f}}{P_{r_{f}}} L_{f} w_{f}=L_{f}^{2} w_{f}-a_{f}^{2} R t_{f} \theta_{f}+a_{f}^{2} R s_{f} s_{f} \\
& -T a_{f} D_{f} \xi_{3_{f}}+\frac{\sigma_{f}}{P_{m_{m}}} D_{f} b_{f}-Q_{f} D_{f}^{2} w_{f} \\
& \frac{\sigma_{f}}{P_{r_{f}}} \xi_{3_{f}}=L_{f} \xi_{3_{f}}+T a_{f} D_{f} w_{f}+D_{f} j_{3_{f}} \\
& \sigma_{f} \theta_{f}+F_{T} w_{f}=L_{f} \theta_{f} \\
& \frac{\sigma_{f}}{L e_{f}} s_{f}+F_{S} w_{f}=L_{f} s_{f} \\
& \frac{\sigma_{f}}{P_{m_{f}}} j_{3_{f}}=Q_{f} D_{f} \xi_{3_{f}}+L_{f} j_{3_{f}} \\
& \frac{\sigma_{f}}{P_{m_{f}}} b_{f}=Q_{f} D_{f} w_{f}+L_{f} b_{f} \\
& -\frac{D a}{\varphi P_{r_{m}}} \sigma_{m} L_{m} w_{f}=L_{m} w_{m}+a_{m}^{2} R t_{m} \theta_{m}-a_{m}^{2} R s_{m} s_{m} \\
& +T a_{m} D \xi_{3_{m}}-\frac{D a}{P_{m_{m}}} \sigma_{m} D_{m} b_{m}+D a Q_{m} D_{m}^{2} w_{m} \\
& -\frac{D a}{\varphi P_{r_{m}}} \sigma_{m} \xi_{3_{m}}=\xi_{3_{m}}-D a L_{m} \xi_{3_{m}} \\
& -T a_{m} D w_{m}-D a D_{m} j_{3_{m}} \\
& G_{m} \sigma_{m} \theta_{m}+F_{T} w_{m}=L_{m} \theta_{m} \\
& \frac{\varphi}{L e_{m}} \sigma_{m} s_{m}+F_{S} w_{m}=L_{m} s_{m} \\
& \frac{\sigma_{m}}{P_{m_{m}}} j_{3_{f} m}=Q_{m} D_{m} \xi_{3_{f m}}+L_{f} j_{3_{m}} \\
& \frac{\sigma_{m}}{P_{m_{m}}} b_{m}=Q_{m} D_{m} w_{m}+L_{m} b_{m}
\end{aligned}
$$

where $a_{f}=\sqrt{n_{f}^{2}+m_{f}^{2}}$ and $a_{m}=\sqrt{n_{m}^{2}+m_{m}^{2}}$ are non- dimensional wave numbers in the fluid layer and porous medium layer respectively, $\sigma$ is the growth rate and

$$
\begin{aligned}
& a_{f}=\hat{d} a_{m}, \sigma_{f}=\frac{\hat{d}^{2}}{\varepsilon_{T}} \sigma_{m}, \\
& D_{f}=\frac{\partial}{\partial x_{3}}, x_{3} \in[0,1], D_{m}=\frac{\partial}{\partial x_{3}}, x_{3} \in[-1,0], \\
& L_{f}=\left(D_{f}^{2}-a_{f}^{2}\right) \text { and } L_{m}=\left(D_{m}^{2}-a_{m}^{2}\right) .
\end{aligned}
$$

The boundary conditions in the final form are

$$
\left.\begin{array}{c}
w_{f}=0, D_{f} w_{f}=0, \theta_{f}=0, \\
s_{f}=0, \xi_{3_{f}}=0, \\
D b_{f}=0, j_{3_{f}}=0
\end{array}\right\} \text { on } x_{3}=1
$$

\section{Numerical Solution}

A Legendre polynomials (see Bukhari [5]) is applied to solve the Equations (59)-(70) with the relevant boundary conditions (71)-(73), and we map $x_{3} \in[0,1]$ and $x_{3} \in[-1,0]$ into $z \in[-1,1]$ by the transformations $z=2 x_{3}-1$ and $z=2 x_{3}+1$ respectively, and get

$$
\frac{\partial}{\partial x_{3}}=2 \frac{\partial}{\partial z} \text {, thus } D_{f}=D_{m}=2 \frac{\partial}{\partial z}=D, z \in[-1,1] \text {. }
$$


then, suppose that

$$
y_{r}(z)=\sum_{k=0}^{M-1} \alpha_{k r} P_{k}(z), 1 \leq r \leq 28 \quad z \in[-1,1]
$$

let the variables $y_{r}$ where $1 \leq r \leq 28$ be defined by

$$
\begin{aligned}
& y_{1}=w_{f}, y_{2}=D_{f} w_{f}, y_{3}=D_{f}^{2} w_{f}, y_{4}=D_{f}^{3} w_{f}, \\
& y_{5}=\xi_{3_{f}}, y_{6}=D_{f} \xi_{3_{f}}, y_{7}=\theta_{f}, y_{8}=D_{f} \theta_{f}, \\
& y_{9}=s_{f}, y_{10}=D_{f} s_{f}, y_{11}=b_{f}, y_{12}=D b_{f} \\
& y_{13}=J_{f}, y_{14}=D J_{f} \\
& y_{15}=w_{m}, y_{16}=D_{m} w_{m}, y_{17}=D_{m}^{2} w_{m}, y_{18}=D_{m}^{3} w_{m}, \\
& y_{19}=\xi_{3_{m}}, y_{20}=D_{m} \xi_{3_{m}}, y_{21}=\theta_{m}, y_{22}=D_{m} \theta_{m}, \\
& y_{23}=s_{m}, y_{24}=D_{m} s_{m}, y_{25}=b_{m}, y_{26}=D b_{m} \\
& y_{27}=J_{m}, y_{28}=D J_{m} .
\end{aligned}
$$

Then the Equations (59)-(70) can be rewritten in a system of twenty ordinary differential equations of first order, since $D_{f}=D_{m}=D$ and if we put $\sigma_{m}=\sigma$ then $\sigma_{f}=\frac{\hat{d}^{2}}{\varepsilon_{T}} \sigma$ so the eigenvalue problem can be reformulated in the form

$$
\frac{\mathrm{d} Y}{\mathrm{~d} z}=\boldsymbol{A} Y+\sigma \boldsymbol{B} Y, \quad z \in[-1,1]
$$

where $\boldsymbol{A}$ and $\boldsymbol{B}$ are real $28 \times 28$ matrices. The final eigenvalue problem reduces to $\boldsymbol{E V}=\sigma \boldsymbol{F V}$ where matrices $\boldsymbol{E}$ and $\boldsymbol{F}$ have the block forms. The boundary conditions replace the $1 M$ th, $2 M$ th, $\cdots 28 M$ th rows of $E$ and $F$.

\section{Results and Remarks}

Using Legendre polynomials, the eigenvalue problems (59)-(70) with the boundary conditions (71)-(73) are transformed to a system of fourteen ordinary differential equations of first order in the fluid layer and a system of fourteen ordinary differential equations of first order in the porous layer with twenty eight boundary conditions. In this work, we will discuss the numerical results through two cases-when the heat and salt concentration affected from above and below.

Case (1): the heat and the salt concentration affected from above.

Here, we put $F_{T}=1, F_{S}=1$ and the value of the initial salt Rayleigh number of the porous medium $R s_{m}=5000$ to find the thermal Rayleigh number of the porous medium $R t_{m}$ corresponding to the wave numbers $a_{m}$ for the different values of $T a_{m}, Q_{m}, D a_{m}, \hat{d} \quad P_{r_{f}}, L e_{f}$ and $P_{m_{f}}$. In this case, the eigenvalues are real, and thus the stationary instability happens, as shown in the fol-

\begin{tabular}{|c|c|c|c|c|}
\hline \multirow{2}{*}{$a_{m}$} & \multicolumn{4}{|c|}{$R t_{m}$} \\
\hline & $Q_{m}=100$ & $Q_{m}=200$ & $Q_{m}=300$ & $Q_{m}=500$ \\
\hline 1 & 1411.45 & 1404.08 & 2383.89 & 1973.94 \\
\hline 2 & 3791.05 & 3792.31 & 4113.01 & 4043.10 \\
\hline 3 & 4244.92 & 4247.22 & 4444.95 & 4428.29 \\
\hline 4 & 4407.32 & 4409.66 & 4563.75 & 4561.69 \\
\hline 5 & 4480.67 & 4482.88 & 4616.37 & 4619.42 \\
\hline 6 & 4516.27 & 4518.33 & 4640.33 & 4645.42 \\
\hline 7 & 4532.33 & 4534.26 & 4649.09 & 4655.00 \\
\hline 8 & 4536.74 & 4538.55 & 4648.52 & 4654.70 \\
\hline 9 & 4533.34 & 4535.04 & 4641.48 & 4647.67 \\
\hline 10 & 4524.16 & 4525.77 & 4629.49 & 4635.56 \\
\hline 11 & 4510.35 & 4511.89 & 4613.41 & 4619.30 \\
\hline 12 & 4492.58 & 4494.05 & 4593.73 & 4599.37 \\
\hline 13 & 4471.24 & 4472.63 & 4570.70 & 4576.11 \\
\hline 14 & 4446.53 & 4447.87 & 4544.49 & 4549.64 \\
\hline 15 & 4418.59 & 4419.87 & 4515.16 & 4520.04 \\
\hline 16 & 4387.44 & 4388.67 & 4482.70 & 4487.33 \\
\hline 17 & 4353.09 & 4354.26 & 4447.09 & 4451.48 \\
\hline 18 & 4315.48 & 4316.60 & 4408.27 & 4412.42 \\
\hline 19 & 4274.55 & 4275.62 & 4366.16 & 4370.05 \\
\hline 20 & 4230.19 & 4231.21 & 4320.64 & 4324.32 \\
\hline
\end{tabular}
lowing Tables 1-4 and Figures 2-5. Therefore, we concluded that:
Table 1. The relation between $a_{m}$ and $R t_{m}$ for different values of $T a_{m}$ when $\hat{d}=0.01, D a_{m}=0.0003, Q_{m}=100$, $P_{r_{f}}=L e_{f}=1$ and $P_{m_{f}}=3$.

\begin{tabular}{cccc}
\hline \multirow{3}{*}{$a_{m}$} & \multicolumn{3}{c}{$R t_{m}$} \\
\cline { 2 - 4 } & $T a_{m}=5$ & $T a_{m}=10$ & $T a_{m}=15$ \\
\hline 1 & 1411.452 & 4404.851 & 15442.275 \\
2 & 3791.053 & 1926.006 & 1612.915 \\
3 & 4244.928 & 3112.021 & 962.857 \\
4 & 4407.321 & 3531.430 & 1870.107 \\
5 & 4480.673 & 3724.639 & 2290.704 \\
6 & 4516.271 & 3826.280 & 2517.540 \\
7 & 4532.339 & 3883.113 & 2651.554 \\
8 & 4536.749 & 3914.926 & 2735.177 \\
9 & 4533.343 & 3931.247 & 2788.741 \\
10 & 4524.163 & 3937.112 & 2822.960 \\
11 & 4510.357 & 3935.370 & 2843.926 \\
12 & 4492.585 & 3927.696 & 2855.233 \\
13 & 4471.240 & 3915.128 & 2859.100 \\
14 & 4446.537 & 3898.286 & 2856.979 \\
15 & 4418.593 & 3877.578 & 2849.797 \\
16 & 4387.447 & 3853.229 & 2838.122 \\
17 & 4353.093 & 3825.368 & 2822.374 \\
18 & 4315.484 & 3794.040 & 2802.753 \\
19 & 4274.550 & 3759.245 & 2779.416 \\
20 & 4230.195 & 3720.946 & 2752.387 \\
\hline
\end{tabular}

Table 2. The relation between $a_{m}$ and $R t_{m}$ for different values of $Q_{m}$ when $T a_{m}=5, \hat{d}=0.01, D a_{m}=0.0003$, $P_{r_{f}}=L e_{f}=1$ and $P_{m_{f}}=3$. 
Table 3. The relation between $\boldsymbol{a}_{m}$ and $R t_{m}$ for different values of $D a_{m}$ when $Q_{m}=100, T a_{m}=5, \hat{d}=0.01, P_{r_{f}}=L e_{f}=1$ and $P_{m_{f}}=3$.

\begin{tabular}{cccc}
\hline & \multicolumn{3}{c}{$R t_{m}$} \\
\cline { 2 - 4 }$a_{m}$ & $D a_{m}=0.000003$ & $D a_{m}=0.00003$ & $D a_{m}=0.003$ \\
\hline 1 & 2199.443 & 2261.610 & 3012.461 \\
2 & 4045.497 & 4064.695 & 4294.012 \\
3 & 4400.439 & 4411.751 & 4544.738 \\
4 & 4527.884 & 4536.455 & 4636.163 \\
5 & 4584.631 & 4591.995 & 4676.610 \\
6 & 4610.809 & 4617.560 & 4693.868 \\
7 & 4620.854 & 4627.285 & 4697.788 \\
8 & 4621.115 & 4627.358 & 4692.409 \\
9 & 4614.721 & 4620.852 & 4679.407 \\
10 & 4603.393 & 4609.440 & 4659.348 \\
11 & 4588.100 & 4594.085 & 4632.211 \\
12 & 4569.475 & 4575.375 & 4597.615 \\
13 & 4547.890 & 4553.683 & 4554.980 \\
14 & 4523.591 & 4529.256 & 4503.524 \\
15 & 4496.759 & 4502.256 & 4442.376 \\
16 & 4467.546 & 4472.792 & 4370.544 \\
17 & 4436.022 & 4440.952 & 4286.951 \\
18 & 4402.228 & 4406.774 & 4190.427 \\
19 & 4366.225 & 4370.297 & 4079.744 \\
20 & 4328.078 & 4331.551 & 3953.576 \\
\hline & & & \\
\hline
\end{tabular}

Table 4. The relation between $\boldsymbol{a}_{m}$ and $R t_{m}$ for different values of $\hat{d}$ when $Q_{m}=100, D a_{m}=0.0003, T a_{m}=5$, $P_{r_{f}}=L e_{f}=1$ and $P_{m_{f}}=3$.

\begin{tabular}{ccccc}
\hline & \multicolumn{4}{c}{$R t_{m}$} \\
\cline { 2 - 5 }$a_{m}$ & $\hat{d}=0.005$ & $\hat{d}=0.1$ & $\hat{d}=0.2$ & $\hat{d}=0.3$ \\
\hline 1 & 2490.38 & 2172.34 & 2075.69 & 2037.71 \\
2 & 4160.48 & 3774.26 & 3637.21 & 3590.73 \\
3 & 4474.34 & 4210.74 & 4146.82 & 4136.76 \\
4 & 4583.48 & 4411.01 & 4383.17 & 4382.40 \\
5 & 4630.30 & 4513.35 & 4500.16 & 4500.78 \\
6 & 4650.60 & 4567.15 & 4560.02 & 4560.55 \\
7 & 4656.94 & 4594.47 & 4590.02 & 4590.30 \\
8 & 4654.70 & 4605.96 & 4602.80 & 4602.90 \\
9 & 4646.47 & 4607.13 & 4604.62 & 4604.60 \\
10 & 4633.62 & 4600.96 & 4598.81 & 4598.71 \\
11 & 4616.88 & 4589.15 & 4587.20 & 4587.04 \\
12 & 4596.69 & 4572.68 & 4570.87 & 4570.64 \\
13 & 4573.27 & 4552.16 & 4550.43 & 4550.15 \\
14 & 4546.74 & 4527.94 & 4526.26 & 4525.93 \\
15 & 4517.15 & 4500.22 & 4498.58 & 4498.20 \\
16 & 4484.48 & 4469.11 & 4467.49 & 4467.07 \\
17 & 4448.69 & 4434.63 & 4433.04 & 4432.57 \\
18 & 4409.72 & 4396.79 & 4395.20 & 4394.70 \\
19 & 4367.48 & 4355.50 & 4353.93 & 4353.40 \\
20 & 4321.85 & 4310.72 & 4309.19 & 4308.56 \\
\hline & & & &
\end{tabular}

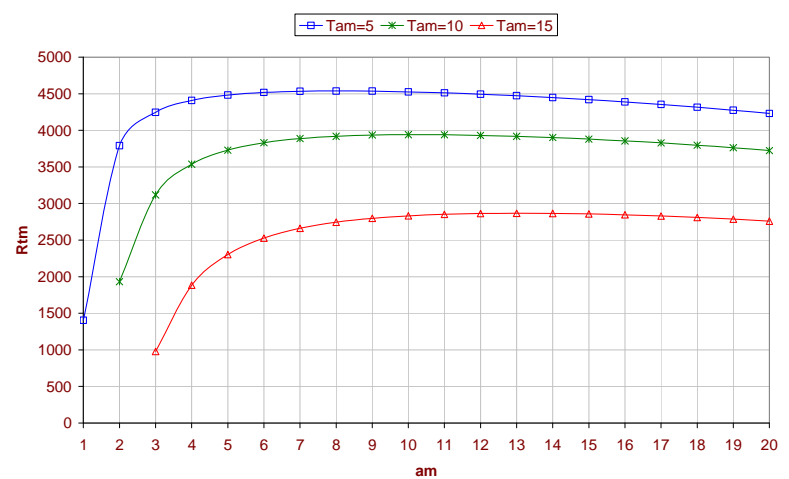

Figure 2. The relation between $a_{m}$ and $R t_{m}$ for different values of $\mathrm{Ta}_{m}$ when $\hat{\boldsymbol{d}}=0.01, D a_{m}=0.0003, Q_{m}=100$, $P_{r_{f}}=L e_{f}=1$ and $P_{m_{f}}=3$.

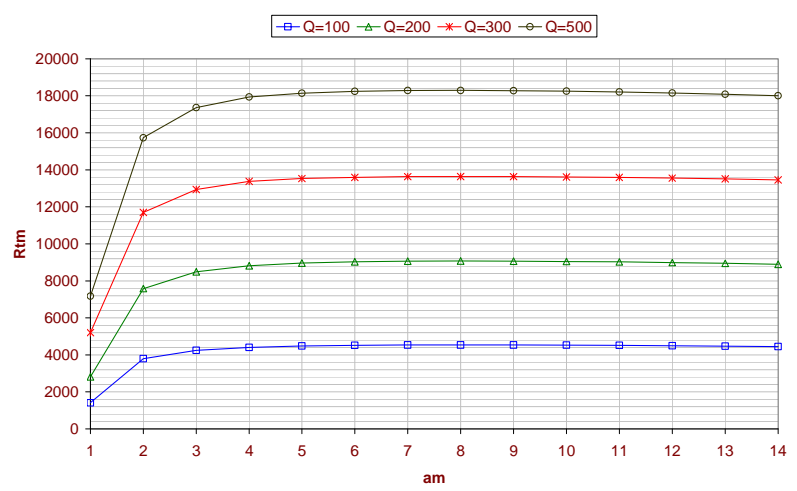

Figure 3. The relation between $a_{m}$ and $R t_{m}$ for different values of $Q_{m}$ when $T a_{m}=5, \hat{d}=0.01, D a_{m}=0.0003$, $P_{r_{f}}=L e_{f}=1$ and $P_{m_{f}}=3$.

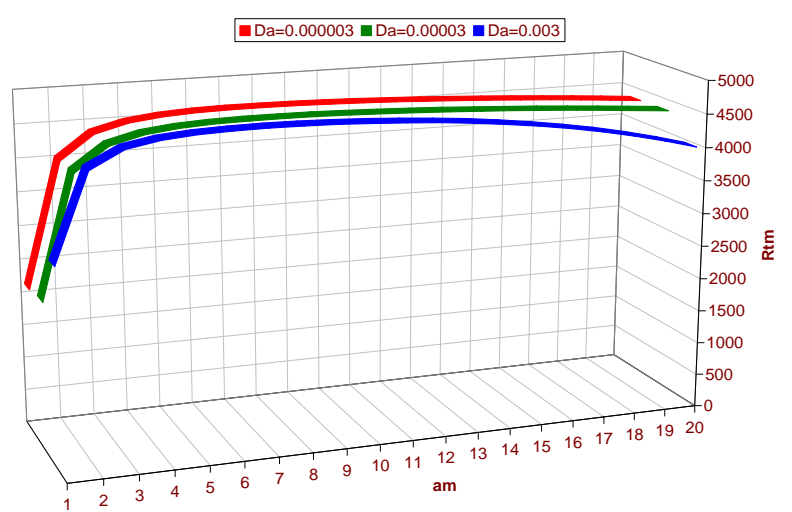

Figure 4. The relation between $a_{m}$ and $R t_{m}$ for different values of $D a_{m}$ when $Q_{m}=100, T a_{m}=5, \hat{d}=0.01$, $\boldsymbol{P}_{r_{f}}=L \boldsymbol{e}_{f}=\mathbf{1}$ and $\boldsymbol{P}_{m_{f}}=\mathbf{3}$.

- As $T a_{m}$ increases $R t_{m}$ decreases which means that the increase of the rotation causes the increase of the thermal convections, leading to an increase in the instability of the fluid, as shown in Figure 2 and its numerical results in Table 1. 


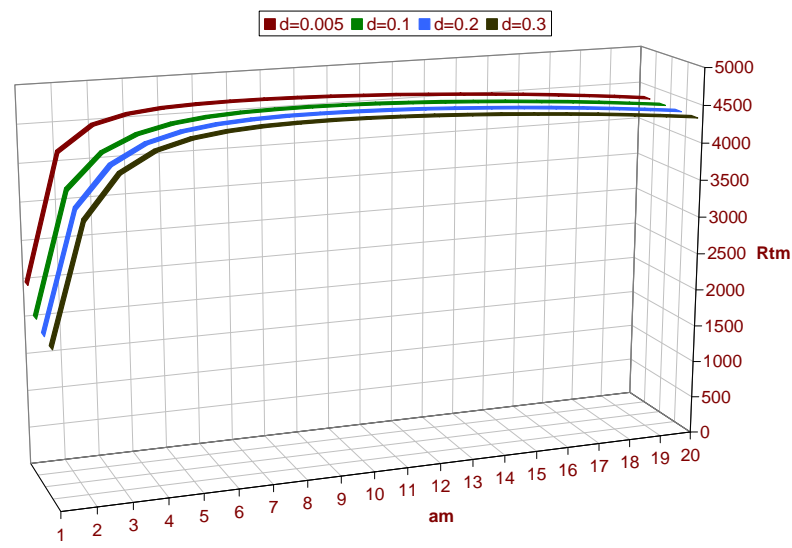

Figure 5. The relation between $a_{m}$ and $R t_{m}$ for different values of $\hat{d}$ when $Q_{m}=100, D a_{m}=0.0003, T a_{m}=5$, $P_{r_{f}}=L e_{f}=1$ and $P_{m_{f}}=3$.

- The presence of the linear magnetic field helps reduce the currents of the thermal convections, meaning that the stability will increase in the fluid, as shown in Figure 3 and its numerical results in Table 2.

- The fluid becomes unstable when the permeability of the porous medium increases, as shown in Figure 4 and its numerical results in Table 3.

- The increase of depth ratio between the two layers makes the fluid unstable meaning that as $\hat{d}$ increases $R t_{m}$ decreases, as shown in Figure 5 and its numerical results in Table 4.

Case (2): the heat and the salt concentration affected from below.

Here, we put $F_{T}=-1, F_{S}=-1$ and the value of the initial salt Rayleigh number of a porous medium $R s_{m}=$ 10000 , to find the thermal Rayleigh numbers of a porous medium, $R t_{m}$ corresponding to wave numbers, $a_{m}$ for different values of $T a_{m}, Q_{m}, D a_{m}, \hat{d} \quad P_{r_{f}}, L e_{f}$ and $P_{m_{f}}$. In this case, the eigenvalues are complex, and thus the overstability happens, as shown in the following Tables 5-8 and Figures 6-9. Therefore, we got the following results:

- The increase of the rotation helps reduce the currents of the thermal convections meaning that the stability will increase in the fluid, as displayed in Figure 6 and its numerical results in Table 5.

- The presence of the linear magnetic field makes the fluid more stable as displayed in Figure 7 and its numerical results in Table 6.

- As $D a_{m}$ increases $R t_{m}$ decreases which means that the increase of the permeability causes the increase of the thermal convections, leading to an increase in the instability of the fluid, as shown in Figure 8 and its numerical results in Table 7.

- As $\hat{d}$ increase $R t_{m}$ increases meaning that the in-
Table 5. The relation between $\boldsymbol{a}_{m}$ and $R t_{m}$ for different values of $\mathrm{Ta}_{m}$ when $\hat{d}=0.2, Q_{m}=100, D a_{m}=0.0003$, $P_{r_{f}}=L e_{f}=1$ and $P_{m_{f}}=3$.

\begin{tabular}{cccc}
\hline \multirow{3}{*}{$a_{m}$} & \multicolumn{3}{c}{$R t_{m}$} \\
\cline { 2 - 4 } & $T a_{m}=0.5$ & $T a_{m}=0.75$ & $T a_{m}=1$ \\
\hline 1 & 307.785 & 349.180 & 395.390 \\
2 & 223.032 & 241.258 & 262.237 \\
3 & 201.231 & 213.226 & 228.383 \\
4 & 190.352 & 198.527 & 209.907 \\
5 & 184.497 & 190.068 & 198.264 \\
6 & 182.855 & 186.774 & 192.528 \\
7 & 185.456 & 188.387 & 192.523 \\
8 & 192.435 & 194.781 & 197.972 \\
9 & 203.924 & 205.910 & 208.575 \\
10 & 220.055 & 221.804 & 224.162 \\
11 & 240.976 & 242.559 & 244.717 \\
12 & 266.869 & 268.330 & 270.341 \\
13 & 297.955 & 299.322 & 301.217 \\
14 & 334.500 & 335.792 & 337.593 \\
15 & 376.815 & 378.047 & 379.769 \\
\hline
\end{tabular}

Table 6. The relation between $\boldsymbol{a}_{m}$ and $R t_{m}$ for different values of $Q_{m}$ when $T a_{m}=0.5, \hat{d}=0.2, D a_{m}=0.001$, $P_{r_{f}}=L e_{f}=1$ and $P_{m_{f}}=3$.

\begin{tabular}{cccc}
\hline$a_{m}$ & \multicolumn{3}{c}{$R t_{m}$} \\
\cline { 2 - 4 } & $Q_{m}=10$ & $Q_{m}=50$ & $Q_{m}=100$ \\
\hline 1 & 300.107 & 303.729 & 307.785 \\
2 & 221.652 & 221.929 & 223.032 \\
3 & 199.023 & 199.797 & 201.231 \\
4 & 187.148 & 188.482 & 190.352 \\
5 & 181.057 & 182.540 & 184.497 \\
6 & 179.641 & 181.046 & 182.855 \\
7 & 182.618 & 183.871 & 185.456 \\
8 & 189.975 & 191.069 & 192.435 \\
9 & 201.789 & 202.743 & 203.924 \\
10 & 218.181 & 219.020 & 220.055 \\
11 & 239.307 & 240.056 & 240.976 \\
12 & 265.357 & 266.035 & 266.869 \\
13 & 296.563 & 297.187 & 297.955 \\
14 & 333.199 & 333.781 & 334.500 \\
15 & 375.583 & 376.134 & 376.815 \\
\hline
\end{tabular}


Table 7. The relation between $\boldsymbol{a}_{m}$ and $R t_{m}$ for different values of $D a_{m}$ when $\mathrm{Ta}_{m}=0.5, Q_{m}=50, \hat{d}=0.2, P_{r_{f}}=$ $L e_{f}=1$ and $P_{m_{f}}=3$.

\begin{tabular}{cccc}
\hline & \multicolumn{3}{c}{$R t_{m}$} \\
\cline { 2 - 4 }$a_{m}$ & $D a_{m}=0.00001$ & $D a_{m}=0.0001$ & $D a_{m}=0.001$ \\
\hline 1 & 331.794 & 326.913 & 307.785 \\
2 & 245.825 & 243.922 & 223.032 \\
3 & 232.947 & 230.599 & 201.231 \\
4 & 233.441 & 229.691 & 190.352 \\
5 & 239.325 & 233.587 & 184.497 \\
6 & 248.566 & 240.404 & 182.855 \\
7 & 260.452 & 249.527 & 185.456 \\
8 & 274.684 & 260.736 & 192.435 \\
9 & 291.119 & 273.963 & 203.924 \\
10 & 309.681 & 289.209 & 220.055 \\
11 & 330.329 & 306.512 & 240.976 \\
12 & 353.042 & 325.946 & 266.869 \\
13 & 377.805 & 347.627 & 297.955 \\
14 & 402.569 & 371.724 & 334.500 \\
15 & 427.332 & 398.437 & 376.815 \\
\hline
\end{tabular}

Table 8. The relation between $a_{m}$ and $R t_{m}$ for different values of $\hat{d}$ when $T a_{m}=0.5, D a_{m}=0.001, Q_{m}=100$, $P_{r_{f}}=L e_{f}=1$ and $P_{m_{f}}=3$.

\begin{tabular}{ccc}
\hline & \multicolumn{2}{c}{$R t_{m}$} \\
\cline { 2 - 3 }$a_{m}$ & $\hat{d}=0.02$ & $\hat{d}=0.2$ \\
\hline 1 & 325.430 & 307.785 \\
2 & 225.413 & 223.032 \\
3 & 203.245 & 201.231 \\
4 & 192.852 & 190.352 \\
5 & 187.424 & 184.497 \\
6 & 186.039 & 182.855 \\
7 & 188.738 & 185.456 \\
8 & 195.706 & 192.435 \\
9 & 207.121 & 203.924 \\
10 & 223.150 & 220.055 \\
11 & 243.962 & 240.976 \\
12 & 269.748 & 266.869 \\
13 & 300.736 & 297.955 \\
14 & 337.192 & 334.500 \\
15 & 379.428 & 376.815 \\
\hline
\end{tabular}

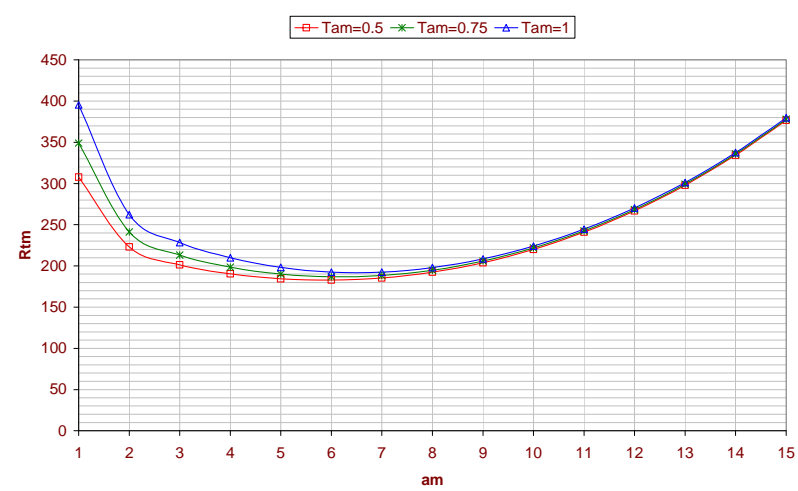

Figure 6. The relation between $\boldsymbol{a}_{\boldsymbol{m}}$ and $\boldsymbol{R t _ { m }}$ for different values of $\mathrm{Ta}_{m}$ when $\hat{d}=0.2, Q_{m}=100, D a_{m}=0.0001$, $P_{r_{f}}=L e_{f}=1$ and $P_{m_{f}}=3$.

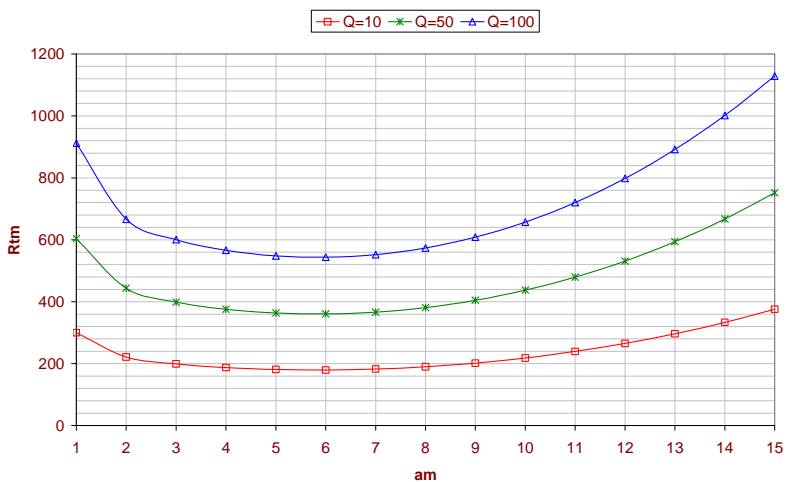

Figure 7. The relation between $a_{m}$ and $R t_{m}$ for different values of $Q_{m}$ when $T a_{m}=0.5, \hat{d}=0.2, D a_{m}=0.001$, $P_{r_{f}}=L e_{f}=1$ and $P_{m_{f}}=3$.

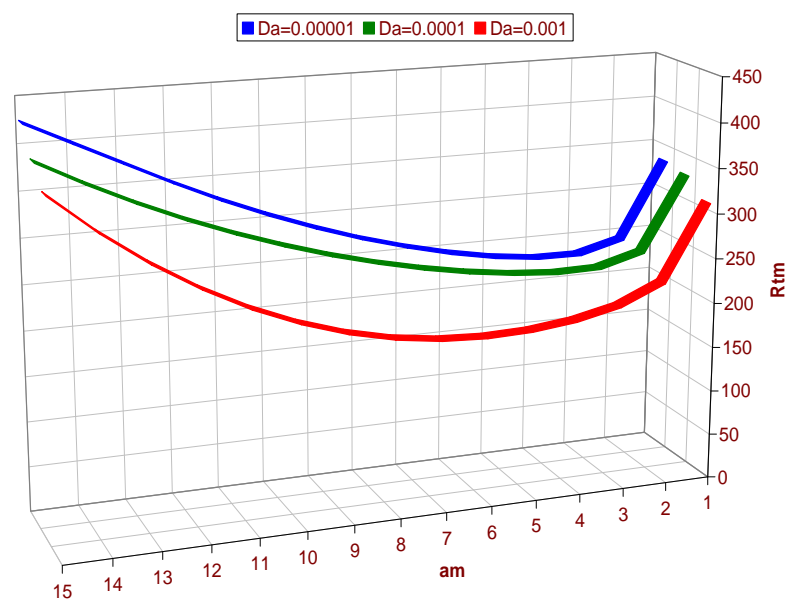

Figure 8. The relation between $\boldsymbol{a}_{\boldsymbol{m}}$ and $\boldsymbol{R} t_{m}$ for different values of $D a_{m}$ when $T_{m}=0.5, Q_{m}=50, \hat{d}=0.2, P_{r_{f}}=$ $L e_{f}=1$ and $P_{m_{f}}=3$.

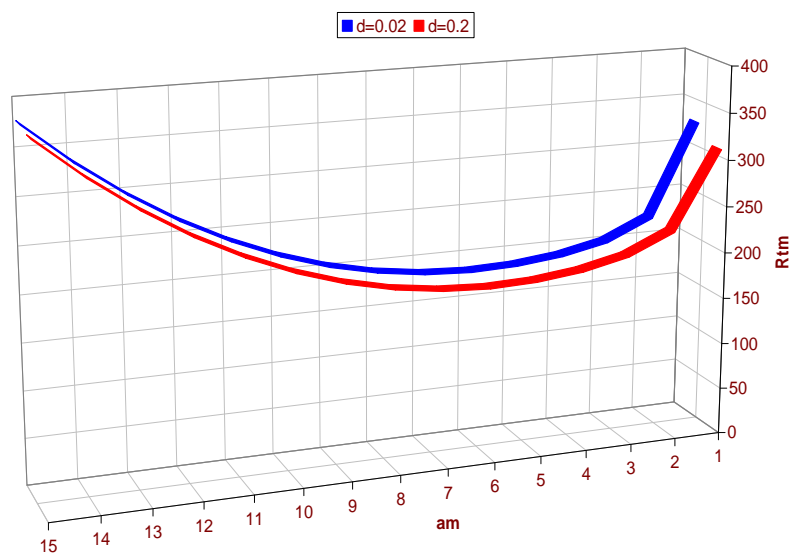

Figure 9. The relation between $\boldsymbol{a}_{m}$ and $R t_{m}$ for different values of $\hat{d}$ when $\mathrm{Ta}_{m}=0.5, D a_{m}=0.001, Q_{m}=100$, $P_{r_{f}}=L e_{f}=1$ and $P_{m_{f}}=3$. 
crease of the depth ratio between the two layers makes the fluid unstable, as displayed in Figure $\mathbf{9}$ and its numerical results in Table 8.

\section{REFERENCES}

[1] H. Benard, "Les Tourbillons Cellulaires Dans une Nappe Liquide," Revue Genral des Sciences Pures et Appliquess, Vol. 11, 1900, pp. 1216-1271, 1309-1328.

[2] H. Benard, "Les Tourbillons Cellulaires Dans une Nappe Liquide Transportant de la Chalaur par Convection en Regime Permanent," Annales de Chimie et de Physique, Vol. 23, 1901, pp. 62-114.

[3] S. Chandrasekhar, "The Instability of a Layer of Fluid Heated below and Subject to Coriolis Forces," Proceedings of the Royal Society A (London), Vol. 217, No. 1130, 1953, pp. 306-327. doi:10.1098/rspa.1953.0065

[4] S. Chandrasekhar and D. D. Elbert, "The Instability of a Layer of Fluid Heated below and Subject to Coriolis Forces. II," Proceedings of the Royal Society A (London), Vol. 231, No. 1185, 1955, pp. 198-210.

[5] D. A. Nield, "Onset of Themohaline Convection in a Porous Medium," Water Resources Research, Vol. 4, No. 3, 1968, pp. 553-560.

[6] J. Taunton and E. Lightfoot, "Thermohaline Instability and Salt Fingers in a Porous Medium," Physics of Fluids, Vol. 15, No. 748, 1972, pp. 784-753.

[7] W. J. Sun, "Convection Instability in Superposed Porous and Free Layers," Ph.D. Dissertation, University of Minnesota, Minneapolis, 1973.

[8] D. A. Nield, "Onset of Convection in a Fluid Layer Overlying a Porous Medium," Journal of Fluid Mechanics,
Vol. 81, No. 3, 1977, pp. 513-522. doi:10.1017/S0022112077002195

[9] H. C. Brinkman, "A Calculation of the Viscous Force Exerted by a Flowing Fluid on a Dense Swarm of Particles," Journal of Applied Sciences Research, Vol. Al. 1947, pp. 27-34.

[10] F. Chen and C. F. Chen, "Onset of Finger Convection in a Horizontal Porous Layer Underlying a Fluid Layer," Journal of Heat Transfer, Vol. 110, No. 2, 1988, pp. 403-409. doi:10.1115/1.3250499

[11] K. A. Lindsay and R. R. Ogden, "A Practical Implementation of Spectral Methods Resistant to the Generation of Spurious Eigenvalues Paper No. 92/23," University of Glasgow, Department of Mathematics Preprint Series, Glasgow, 1992

[12] C. J. Lamb, "Hydromagnetic Instability in the Earth's Core," Ph.D. Thesis, Department of Mathematics, University of Glasgow, Glasgow, 1994.

[13] A. F. Bukhari, "A Spectral Eigenvalue Method for MultiLayered Continuua," Ph.D. Thesis, University of Glasgow, Glasgow, 1996.

[14] B. Straughan, "Surface-Tension-Driven Convection in a Fluid Overlying a Porous Layer," Journal of Computational Physics, Vol. 170, No. 1, 2001, pp. 320-337. doi:10.1006/jcph.2001.6739

[15] B. Straughan, "Effect of Property Variation and Modelling on Convection in a Fluid Overlying a Porous Layer," International Journal for Numerical and Analytical Methods in Geomechanics, Vol. 26, No. 1, 2002, pp. 75-97. doi:10.1002/nag.193

[16] F. Allehiany, "Benard Convection in a Horizontal Porous Layer Permeated by a Conducting Fluid in the Presence of Magnetic Field and Coriolis Forces," M.Sc. Thesis, Umm Al-Qura University, Mecca, 2003. 